\title{
An Adaptive Fusion Estimation Algorithm for State of Charge of Lithium-ion Batteries Considering Wide Operating Temperature and Degradation
}

\author{
Xing Shu ${ }^{1}$, Guang $\mathrm{Li}^{2}$, Jiangwei Shen ${ }^{1}$, Wensheng Yan $^{1}$, Zheng Chen ${ }^{1,2^{*}}$ and Yonggang $\mathrm{Liu}^{3 *}$ \\ ${ }^{1}$ Faculty of Transportation Engineering, Kunming University of Science and Technology, Kunming, 650500, China \\ ${ }^{2}$ School of Engineering and Materials Science, Queen Mary University of London, London, E1 4NS, United Kingdom \\ ${ }^{3}$ State Key Laboratory of Mechanical Transmissions \& School of Automotive Engineering, Chongqing University, \\ Chongqing, 400044, China \\ Email: shuxing92@163.com, g.li@qmul.ac.uk, shenjiangwei6@163.com, yanwensheng65@sina.com, \\ chen@kust.edu.cn, andylyg@umich.edu \\ Corresponding Author: Zheng Chen (chen@kust.edu.cn) and Yonggang Liu (andylyg@umich.edu)
}

\begin{abstract}
In this paper, an adaptive fusion algorithm is proposed to robustly estimate the state of charge of lithium-ion batteries. An improved recursive least square algorithm with a forgetting factor is employed to identify parameters of the built equivalent circuit model, and the least square support vector machine algorithm is synchronously leveraged to estimate the battery state of health. On this basis, an adaptive H-infinity filter algorithm is applied to predict the battery state of charge and to cope with uncertainty of model errors and prior noise evaluation. The proposed algorithm is comprehensively validated within a full operational temperature range of battery and with different aging status. Experimental results reveal that the maximum absolute error of the fusion estimation algorithm is less than $1.2 \%$, manifesting its effectiveness and stability when subject to internal capacity degradation of battery and operating temperature variation.
\end{abstract}

Key Words: adaptive H-infinity filter, least square support vector machine, model-based method, state of charge.

NOMENCLATURE

Abbreviations

\begin{tabular}{|c|c|c|c|}
\hline EVs & electric vehicles & RLS-EKF & recursive least square-extended Kalman filter \\
\hline LIBs & lithium-ion batteries & NC-EKF & noise compensation-extended Kalman filter \\
\hline BMS & battery management system & AUKF & adaptive unscented Kalman filter \\
\hline SOC & state of charge & RLS & recursive least square \\
\hline $\mathrm{SOH}$ & state of health & AEKF & adaptive extended Kalman filter \\
\hline SOP & state of power & FF-RLS & recursive least square with forgetting factor \\
\hline SOE & state of energy & AHIF & adaptive $\mathrm{H}$-infinity filter \\
\hline SOF & state of function & LS-SVM & least squares support vector machine \\
\hline $\mathrm{OCV}$ & open circuit voltage & GA & genetic algorithm \\
\hline $\mathrm{ECM}$ & equivalent circuit model & RMSE & root-mean-square error \\
\hline $\mathrm{PF}$ & particle filter & SVM & support vector machine \\
\hline
\end{tabular}




\begin{tabular}{|c|c|c|c|}
\hline $\mathrm{KF}$ & Kalman filter & $\mathrm{RBF}$ & radial basis kernel \\
\hline EKF & extended Kalman filter & $\mathrm{CC}$ & constant current \\
\hline CKF & cubature Kalman filter & $\mathrm{CC}-\mathrm{CV}$ & constant current-constant voltage \\
\hline UKF & unscented Kalman filter & MSE & mean square error \\
\hline HIF & H-infinity filter & UDDS & urban dynamometer driving schedule \\
\hline DEKF & dual extend Kalman filter & GA-AHIF & genetic algorithm based adaptive $\mathrm{H}$-infinity filter \\
\hline \multicolumn{4}{|l|}{ Symbols } \\
\hline$V_{O C V}$ & open circuit voltage & $T$ & battery temperature \\
\hline$R_{0}$ & internal ohmic resistance & $V_{\text {test }}$ & measurement voltage \\
\hline$R_{1}$ & polarization resistance & $N$ & test time \\
\hline$C_{1}$ & polarization capacitance & $u_{k}$ & input variable \\
\hline$E$ & terminal voltage & $x_{k}$ & state variable \\
\hline$I$ & loading current & $y_{k}$ & measurement variable with noise \\
\hline$V_{0}$ & ohmic voltage & $z_{k}$ & linear combination of estimation states \\
\hline$V_{1}$ & polarization voltage & $L_{k}$ & unit matrix \\
\hline$s_{k}$ & state of charge at sampling $k$ & $w_{k}$ & system noise \\
\hline$\eta$ & coulomb efficiency & $v_{k}$ & measurement noise \\
\hline$\Delta t$ & sampling interval time & $P_{0}$ & symmetric positive matrices \\
\hline$Q_{n}$ & battery rated capacity & $Q_{k}$ & symmetric positive matrices \\
\hline$w_{1}$ & process noise for state of charge & $R_{k}$ & symmetric positive matrices \\
\hline$w_{2}$ & $\begin{array}{l}\text { process noise for polarization } \\
\text { voltage }\end{array}$ & $S_{k}$ & symmetric positive matrices \\
\hline$v$ & measurement noise & $J$ & cost function for $\mathrm{H}$-infinity filter \\
\hline$G(s)$ & transformation function & $\varepsilon$ & user-specified boundary value \\
\hline$a_{i}$ & $\begin{array}{l}\text { coefficients associated with the } \\
\text { model parameters }\end{array}$ & $\xi$ & deviation vector \\
\hline $\boldsymbol{\Phi}_{k}$ & input data matrix & $C$ & weight of support vector machine \\
\hline $\boldsymbol{\theta}_{k}$ & parameter matrix & $L$ & Lagrange function \\
\hline $\boldsymbol{K}_{F F-R L S}$ & gain matrix of FF-RLS & $\alpha_{i}$ & Lagrange multiplier \\
\hline $\boldsymbol{P}_{F F-R L S, k}$ & covariance matrix of FF-RLS & $K\left(\boldsymbol{x}, \boldsymbol{x}_{i}\right)$ & kernel function \\
\hline$w$ & white noise information & $V_{\text {start }}$ & starting recode voltage \\
\hline$\lambda$ & forgetting factor & $V_{\text {stop }}$ & ending recode voltage \\
\hline$p_{i}$ & fitting coefficients of OCV & & \\
\hline
\end{tabular}




\section{INTRODUCTION}

Transportation electrification can effectively mitigate environmental pollution and greenhouse gas emission incurred by massive combustion of fossil fuels [1]. Electric vehicles (EVs), representing an important class of vehicles for development, have attracted wide attention due to their zero emission, high efficiency and superior driving performances [2]. Currently, most of EVs are equipped with lithium-ion batteries (LIBs) for storage of electricity $[3,4]$. To guarantee safe efficient operation of batteries, a serviceable battery management system (BMS) is indispensable [5]. The main task of BMS includes accurate measure of battery current, voltage and temperature and on this basis, estimation and evaluation of the inner status [6], i.e., state of $X$, where $X$ can be charge (SOC) [7], health (SOH) [8, 9], power (SOP) [10], energy (SOE) and function (SOF) [11]. Amongst them, SOC refers to the ratio of remaining available capacity over the nominal capacity and directly correlates with current, temperature and terminal voltage of LIBs. Its effective estimation is vital to supply the reference for estimation of remaining driving mileage and avoid abuse operation (over-charge/ discharge). Hence, the estimation algorithm needs to be accurate, quick convergent, reliable and robust [3], which represents a challenging task.

To now, a variety of advanced methods have been proposed, applied and validated to achieve the SOC estimation. Typical ones include coulomb counting, open circuit voltage (OCV) based calibration, data driven and model based algorithms [12]. The coulomb counting method estimates SOC by directly integrating the currents flowing into and out of the batteries over time. Obviously, it is simple and easy to implement in practice, and yet difficult to guarantee estimation accuracy, because it is easily disturbed by measurement error and noise of current, and particularly depends heavily on the initial SOC value [13]. The OCV-based method can obtain SOC accurately with the offline calibrated relationship between OCV and SOC. Apparently, it is not applicable for real-time application, since it is almost not possible to acquire the OCV online. The data driven based method directly extracts internal characteristics of the battery by means of a large amount of operation data, from which the nonlinear mapping relationship between SOC and feature variables is established. The above methods do not require deep understanding of battery operation and inner electrochemical reaction characteristics. However, the estimation accuracy depends largely on selection of feature parameters as well as quality and quantity of training data [14]. The model-based estimation methods are extensively accepted because of their high precision, noise elimination and independence of the initial value. Additionally, they sufficiently merge external 
measurement of voltage, current and temperature and refer to the offline calibration test and model establishment $[7,15]$. To apply model-based algorithms, it is necessary to model dynamic and static electrical characteristics of batteries. Consequently, popular manners, such as equivalent circuit model (ECM) [16], complex electrochemical model [17] and pseudo single particle model [18], are elaborated and applied. Then, typical filters are harnessed to improve the observation precision, including particle filter (PF) [19], Kalman filter (KF) and its extensions such as extend KF (EKF) [20], adaptive EFK (AEKF) [21], cubature KF (CKF) [22], and unscented KF (UKF) [23], nonlinear observer [24], and H-infinity filter (HIF) [25]. In [26], three SOC methods including the dual EKF (DEKF), recursive least square (RLS)-EKF (RLS-EKF) and noise compensation-EKF (NC-EKF) are systemically compared and evaluated. Numerical results manifest that the DEKF and NC-EKF are more robust than RLS-EKF when magnitude of noises aggravates, and the RLS-EKF and NC-EKF features the least and highest computation intensity among these filters, respectively. In [27], an adaptive UKF (AUKF) is leveraged to estimate SOC of the battery module grouped by cells in series connection. Compared with UKF and EKF, the experimental results reveal that the improved AUKF method can track the reference SOC and exhibit high robustness when the process and measurement statistics noises vary stochastically. In [28], an augmented battery model is divided into a SOC sub-model and a resistance-capacitance (RC) circuit sub-model to reduce the cross interference between SOC and voltage of the RC network. The experimental results demonstrate the efficacy of reducing oscillation of SOC and decreasing estimation errors.

In practice, batteries operate within a wide temperate range (usually $-20{ }^{\circ} \mathrm{C}$ to $50{ }^{\circ} \mathrm{C}$ ), and in this context, the model parameters of battery may change with temperature in a nonlinear manner. Similarly, performance degradation of batteries with cycling also leads to variation of model parameters. To address these variations, many efforts have been made to online update model parameters and to cope with external varying conditions and internal parameter variation [26]. In [29], a temperature compensated model is built, and the EKF is investigated to improve the estimation precision of SOC. Ref. [30] analyzes the relationship between the differential voltage (DV) and incremental capacity (IC) in a narrow scope and achieves the simultaneous online estimation of battery capacity and SOC. In [31], a joint algorithm incorporating RLS and AEKF is leveraged to identify the battery parameters and estimate the SOC simultaneously. In addition, the battery capacity is predicted by the Elman neural network in real-time. The numerical results highlight that the estimation error of SOC is less than $2 \%$ at room temperature with different aging status. Nonetheless, the wide operating 
temperature range is not explicitly taken into account, and the maximum estimation error can reach as high as $6 \%$ when the temperature is beyond the normal range. In [32], a Thevenin electric model integrating temperature compensation is introduced and the model parameters are identified by the RLS method under the conditions of different temperatures. In [25], a multi-scale dual HIF is proposed to estimate the battery SOC and capacity simultaneously. However, the operating temperature is not fully considered and the estimation performance is not evaluated when the temperature varies.

As discussed above, even significant contributions have been made to improve estimation accuracy of the SOC in the whole operation range of battery, there still exists a certain room for further improvement when fully considering temperature variation and battery degradation. Motivated by this fact, an advanced fusion estimation algorithm for LIBs is developed based on the ECM considering the whole operating temperature and capacity degradation. First, the battery parameters are identified by an improved RLS with a forgetting factor (FF-RLS) for adaption to environmental temperature variation and capacity degradation. On this basis, the battery SOC is estimated by the adaptive HIF (AHIF) algorithm to cope with the interference of system variation and measurement noise. Furthermore, the least square support vector machine (LS-SVM) is simultaneously employed to estimate the battery $\mathrm{SOH}$, thus assisting the estimation of SOC. The experimental results in terms of capability and operating temperature demonstrate that the algorithm outperforms other commonly used model based methods, such as AEKF. Moreover, the algorithm together with the built ECM is still effective at low temperature and with degraded capacity. This is of great significance for SOC estimation when the battery ages.

The remainder of this paper is structured as follows. In Section II, the ECM and model parameter estimation for LIBs are presented. Section III introduces the adaptive fusion algorithm for the SOC and SOH estimation. Section IV presents and discusses the validation results. Finally, the concluding remarks and future work are given in Section V.

\section{LITHIUM-ION BATTERY MODEL AND PARAMETERS}

\section{A. Lithium-ion Battery Cell Model}

Three broad categories of battery models, including ECM, data driven model and electrochemical model, are successfully introduced to characterize the battery electrical performance. Among these models, the ECM can not only capture the battery's dynamic and static characteristics with preferable precision, but also feature low computation intensity, thereby enabling online real-time application [27]. Thus, in this study, the ECM, as 
depicted in Fig. 1 (a), is employed to describe the electrical behavior of battery. The SOC is expressed as the percentage of remaining capacity over the rated discharging capacity, as:

$$
s_{k+1}=s_{k}-\eta I_{k} \Delta t / Q_{n}
$$

where $s_{k}$ represents the battery SOC at the sampling time $k ; \eta$ denotes the coulomb efficiency; $\Delta t$ represents the sampling time (unit: second), and $Q_{n}$ is the battery rated capacity with the unit of Ampere-hour. In this study, the target LIBs are 21700 LiNMC battery cells and the detailed specification is listed in Table I.

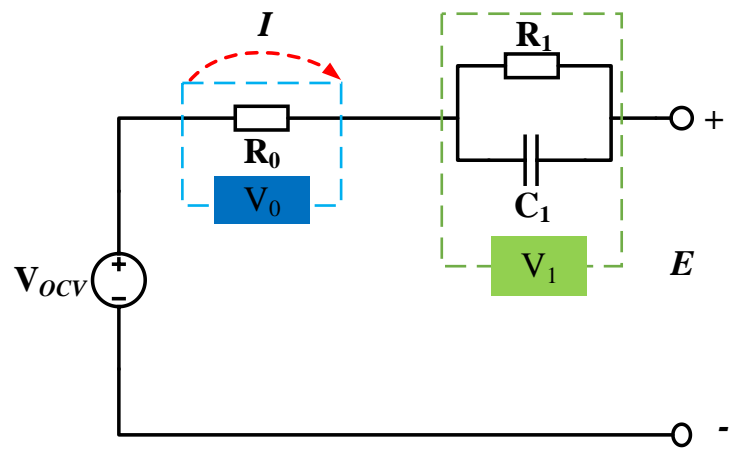

(a)

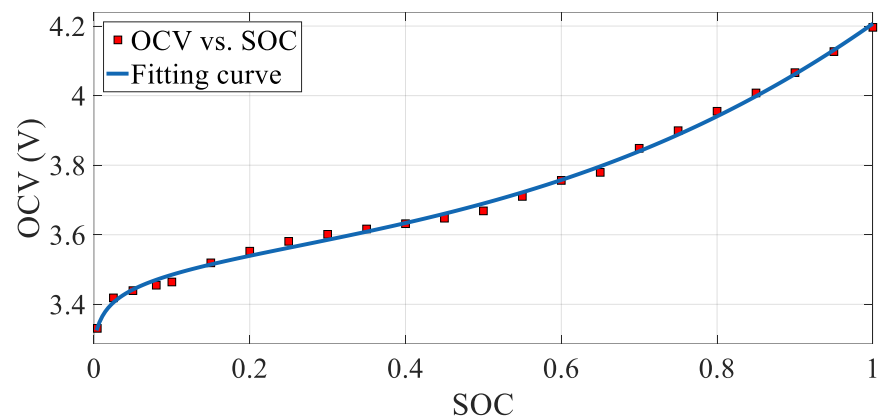

(c)

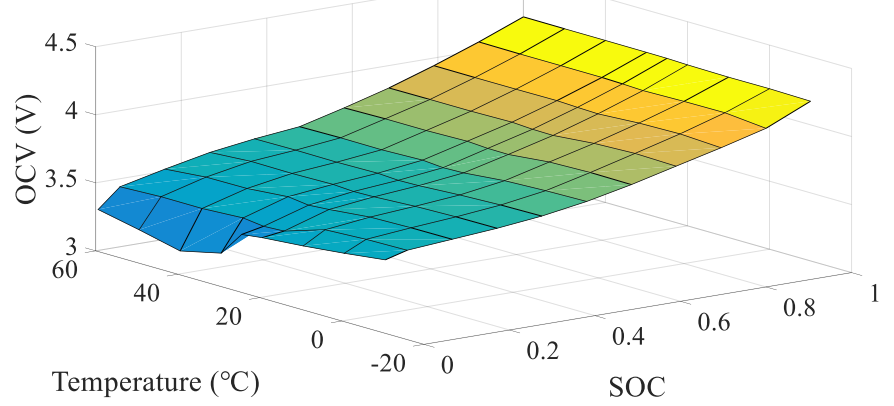

(b)

Fig. 1. The first-order RC model and introduction of related parameters. (a) Equivalent circuit diagram of the battery model; (b) The 3D OCV response surface; (c) The SOC-OCV correlation and fitting results; (d) The Initial capacity at different temperatures.

Table I Specification of the test battery

\begin{tabular}{ll}
\hline Items & Specification \\
\hline Cathode material & $\mathrm{Li}(\mathrm{NiCoMn}) \mathrm{O}_{2}$ \\
Anode material & Graphite \\
Nominal capacity & $4 \mathrm{Ah}$ \\
Allowed operating range of voltage & $2.75-4.2 \mathrm{~V}$ \\
Rated voltage & $3.65 \mathrm{~V}$ \\
Allowed charging temperature & $0-45{ }^{\circ} \mathrm{C}$ \\
Allowed discharging temperature & $-20-60{ }^{\circ} \mathrm{C}$ \\
\hline
\end{tabular}

According to Fig. 1 (a), the following equations can be formulated, as: 


$$
\begin{aligned}
& \left\{\begin{array}{l}
\dot{s}=-\frac{1}{Q_{n}} I_{t}+w_{1} \\
\dot{V}_{1}=-\frac{1}{R_{1} C_{1}} V_{1}+\frac{1}{C_{1}} I_{t}+w_{2}
\end{array}\right. \\
& E=V_{o c}(s, T)-V_{1}-R_{0} I_{t}+v
\end{aligned}
$$

where $V_{O C V}$ denotes the ideal OCV, $R_{0}$ is the internal ohmic resistance, $R_{1}$ and $C_{1}$ are respectively the polarization resistance and capacitance, $E$ is the terminal voltage, and $I$ denotes the loading current. $V_{0}$ and $V_{1}$ represent the ohmic voltage and polarization voltage across $R_{0}$ and $R_{1} ; \quad v$ represents the measurement noise; and $w_{1}$ and $w_{2}$ denote the process noise for SOC and $V_{1}$, respectively.

\section{B. Parameters Acquisition}

Given the strong nonlinear time-varying characteristics of LIBs, it is imperative to construct an online parameter identification algorithm for sufficiently responding to the battery electrical performance. Additionally, the offline identification algorithm is also beneficial to quantitatively describe the relationship between OCV and SOC, temperature and initial capacity. To attain it, the genetic algorithm (GA) is employed to achieve the offline identification algorithm.

\section{1). Online Identification}

According to (2), the state space equation of battery model can be discretized as:

$$
\left\{\begin{array}{l}
V_{1, k}=e^{-\frac{\Delta t}{R_{1} \mid \bar{C}}} V_{1, k-1}+\left(1-e^{-\frac{\Delta t}{R_{1} C_{1}}}\right) R_{1} I_{k-1} \\
E_{k}=V_{o c, k}-V_{1, k}-R_{0} I_{k}
\end{array}\right.
$$

where $E_{k}, V_{1, k}$ and $V_{O C V, k}$ respectively denote the terminal voltage, polarization voltage and OCV at the $k$ th sampling time, and $I_{k}$ is the current at the $k$ th sampling time. Then, from (4), we can get:

$$
E_{k}=V_{O C V, k}-\left(e^{-\frac{\Delta t}{R_{1} I_{1}}} V_{1, k}+\left(1-e^{-\frac{\Delta t}{R_{1} C_{1}}}\right) R_{1} I_{k-1}\right)-I_{k} R_{0}
$$

By eliminating $V_{1, k}$, we can attain:

$$
E_{k}-V_{O C V, k}=e^{-\frac{\Delta t}{R_{1} C_{1}}}\left(E_{k-1}-V_{O C V, k-1}\right)+\left(-R_{0}\right) I_{k}+\left(e^{-\frac{\Delta t}{R_{1} C_{1}}} R_{0}-\left(1-e^{-\frac{\Delta t}{R_{1} C_{1}}}\right) R_{1}\right) I_{k}
$$

Here, we assume that the OCV at the $k$ th step, $V_{O C V, k}$ is equal to $V_{O C V, k-1}$ at the $(k-1)$ th step. Then, the discretization calculation can be rewritten as:

$$
E_{k}=a_{1} V_{O C V, k}+a_{2} E_{k-1}+a_{3} I_{k}+a_{4} I_{k-1}
$$


where $a_{i}(i=1,2,3,4)$ express the coefficients. Consequently, we can attain:

$$
\left\{\begin{array}{l}
y_{k}=\boldsymbol{\Phi}_{k} \boldsymbol{\theta}_{k}+w \\
\boldsymbol{\Phi}_{k}=\left[\begin{array}{llll}
1 & E_{k-1} & I_{k} & I_{k-1}
\end{array}\right] \\
\boldsymbol{\theta}_{k}=\left[\begin{array}{llll}
a_{1} & a_{2} & a_{3} & a_{4}
\end{array}\right]^{T} \\
y_{k}=E_{k}
\end{array}\right.
$$

where $w$ denotes the white noise from the measurement, and

$$
\left\{\begin{array}{l}
a_{1}=\left(1-a_{2}\right) V_{O C V} \\
a_{2}=e^{-\Delta t /\left(R_{1} C_{1}\right)} \\
a_{3}=-R_{0} \\
a_{4}=e^{-\Delta t /\left(R_{1} C_{1}\right)} R_{0}-\left(1-e^{-\Delta t /\left(R_{1} C_{1}\right)}\right) R_{1}
\end{array}\right.
$$

From (9), the ECM parameters can be obtained, as:

$$
\left\{\begin{array}{l}
R_{0}=-a_{3} \\
R_{1}=\frac{a_{2} a_{3}+a_{4}}{a_{2}-1} \\
C_{1}=\frac{\left(1-a_{2}\right) \Delta t}{\left(a_{2} a_{3}+a_{4}\right) \log \left(a_{2}\right)} \\
V_{O C V}=\frac{a_{1}}{1-a_{2}}
\end{array}\right.
$$

As mentioned above, the battery model parameters are easily influenced by the uncertain operation environment. The RLS algorithm based on adaptive filtering can compensate uncertainty of model parameters through recursive parameter correction, so as to accurately capture real-time characteristics of the system. However, for heuristic systems with slow continuous changes, the traditional RLS algorithm is difficult to track variation and provide reliable estimation results [33]. To tackle this issue, the FF-RLS is proposed to realize reliable estimation of model parameters [34]. The main solution steps of FF-RLS are summarized as follows: 1): Input data matrix and initialize parameter matrix

$$
\left\{\begin{array}{l}
\boldsymbol{\Phi}_{k}=\left[\begin{array}{llll}
1 & E_{k-1} & I_{k} & I_{k-1}
\end{array}\right] \\
\boldsymbol{\theta}_{0}=\left[\begin{array}{llll}
a_{1 \_0} & a_{2 \_0} & a_{3 \_0} & a_{4 \_0}
\end{array}\right]^{T}
\end{array}\right.
$$

2): Determine the gain matrix and the covariance matrix

$$
\left\{\begin{array}{l}
\boldsymbol{K}_{F F-R L S, k}=\frac{\boldsymbol{P}_{F F-R L S, k-1} \boldsymbol{\Phi}_{k}^{T}}{\lambda+\boldsymbol{\Phi}_{k} \boldsymbol{P}_{F F-R L S, k-1} \boldsymbol{\Phi}_{k}^{T}} \\
\boldsymbol{P}_{F F-R L S, k}=\frac{\boldsymbol{P}_{F F-R L S, k-1}-\boldsymbol{K}_{F F-R L S, k} \boldsymbol{\Phi}_{k} \boldsymbol{P}_{F F-R L S, k-1}}{\lambda}
\end{array}\right.
$$


where $\lambda$ denotes the forgetting factor, and its function is to strengthen the role of new data and gradually weaken the old data. Usually, $\lambda$ is set within $[0.95,1]$. When $\lambda$ equals 1 , the algorithm degenerates to the conventional RLS.

3): Update the estimation parameters, as:

$$
\hat{\boldsymbol{\theta}}_{k}=\hat{\boldsymbol{\theta}}_{k-1}+\boldsymbol{K}_{F F-R L S}\left(y_{k}-\boldsymbol{\Phi}_{k} \hat{\boldsymbol{\theta}}_{k-1}\right)
$$

4): $k=k+1$ and go to step 1).

It is worth noting that although the FF-RLS can identify the battery parameters under the condition of rapid current excitation, unchanged or tiny varying current excitation still affect precise parameter identification [35]. However, in practice, long time rest and constant current (CC) charging are inevitable. Thus, the current profiles need to be sufficiently diverse to achieve the battery extreme performance. As such, additional iteration process is designed in this paper to solve this problem. In the case of low current or CC, the FF-RLS is temporarily terminated and new parameters are obtained through the weighted average of historical model parameters. The iterative process is designed as:

$$
\hat{\boldsymbol{\theta}}_{k, \text { average }}=\left(1-\frac{1}{\delta}\right) \hat{\boldsymbol{\theta}}_{k-1, \text { average }}+\hat{\boldsymbol{\theta}}_{k} \frac{1}{\delta}
$$

where $\delta=\left(1-\lambda^{k}\right) /(1-\lambda)$. By adding a parameter update loop, the parameter identification method can be applied in any battery driving cycles.

\section{2). Offline Identification}

To acquire the characteristic of the temperature-SOC-OCV model, a group of cells are tested at different operating temperatures with the specially designed current excitation. The experiment temperature is set to $20{ }^{\circ} \mathrm{C}$ to $50{ }^{\circ} \mathrm{C}$ with an interval of $10{ }^{\circ} \mathrm{C}$. During the experiment, the voltage, current and temperature are recorded and the sampling frequency is set to $1 \mathrm{~Hz}$. All the cells are charged and discharged with the current rates of $6 \mathrm{~A}(1.5 \mathrm{C}$, where $\mathrm{C}$ denotes the rated capacity of battery with the unit of Ampere-hour) and $8 \mathrm{~A}(2 \mathrm{C})$. The extracted OCV map at different temperatures and SOC values is depicted in Fig. 1 (b). It can be found that the OCV at different temperatures is not obvious when the SOC is greater than $20 \%$. Here, by referring to the polynomial electrochemical equation introduced in [34], the relationship between OCV and SOC is formulated in (15), and the resulting curve at room temperature is plotted in Fig. 1 (c).

$$
f_{o c v}=p_{0}+p_{1} s+p_{2} s^{2}+p_{3} s^{3}+p_{4} / s+p_{5} \ln (s)+p_{6} \ln (1-s)
$$


where $p_{i}(i=0,1, \ldots, 6)$ are fitting coefficients. The battery initial maximum discharging capacity under different temperatures is plotted in Fig. 1 (d), and the relationship between maximum discharging capacity and temperature can be fitted as:

$$
Q_{n}(T)=-1.681 \times 10^{-07} \cdot T^{4}+1.877 \times 10^{-05} \cdot T^{3}-0.0009316 \cdot T^{2}+0.03541 \cdot T+3.338
$$

where $T$ denotes the battery temperature. To further evaluate performance of the online identification method, the commonly employed offline parameter identification method, i.e., GA, is applied to identify other parameters, including the ohmic resistance, polarization resistance and polarization capacitor [21, 36-38]. The root-mean-square error (RMSE) between the model output and measured voltage is selected as the fitness function:

$$
R M S E=\sqrt{\frac{1}{N} \sum_{i=1}^{N}\left(E-V_{t e s t}\right)^{2}}
$$

where $V_{\text {test }}$ denotes the measurement voltage, and $N$ represents the test time.

\section{SOC AND SOH ESTIMATION}

In this study, the joint estimation includes the SOC and SOH. Actually, both variables are strongly coupled, and precise SOC estimation relies on accurate $\mathrm{SOH}$ value.

\section{A. The SOC Estimation Algorithm}

The KF method assumes that the statistical characteristics of noise are known in advance [23]; however, it is difficult to obtain variance of noise in practice. In addition, the model errors incurred during the modeling process also deteriorates the estimation accuracy. To circumvent limitation of KF and uncertainty of model error and improves the robustness of estimation, the HIF algorithm is employed to estimate the battery SOC. We employ a standard linear time-varying discrete system:

$$
\left\{\begin{array}{l}
x_{k+1}=F_{k} x_{k}+w_{k} \\
y_{k}=H_{k} x_{k}+v_{k} \\
z_{k}=L_{k} x_{k}
\end{array}\right.
$$

where $x_{k}$ is the state variable, $y_{k}$ is measurement variable with noise, and $z_{k}$ represents the linear combination of estimation states. $L_{k}$ is a user-specified matrix, which is set to unit matrix in this paper, $w_{k}$ 
and $v_{k}$ denote the system noise and measurement noise, respectively. The cost function of the HIF can be defined as:

$$
J=\frac{\sum_{k=0}^{N-1}\left\|z_{k}-\hat{z}_{k}\right\|}{\left\|x_{0}-\hat{x}_{0}\right\|_{P_{0}^{-1}}^{2}+\sum_{k=0}^{N-1}\left(\left\|w_{k}\right\|_{Q_{k}^{-1}}^{2}+\left\|v_{k}\right\|_{R_{k}^{-1}}^{2}\right)}
$$

where $P_{0}, Q_{k}, R_{k}$ and $S_{k}$ are positive definite matrices. Intuitively, it is difficult to minimize $J$, and for ease of finding its minimum value, a boundary condition is imposed to assist search of the suboptimal value, as:

$$
J<\varepsilon
$$

where $\varepsilon$ denotes the user-specified boundary value. By incorporating (19) and (20), the boundary condition can be rewritten as:

$$
J=-\varepsilon\left\|x_{0}-\hat{x}_{0}\right\|_{P_{0}^{-1}}^{2}+\sum_{k=0}^{N-1}\left(\left\|w_{k}\right\|_{Q_{k}^{-1}}^{2}+\left\|v_{k}\right\|_{R_{k}^{-1}}^{2}\right)-\varepsilon \sum_{k=0}^{N-1}\left\|z_{k}-\hat{z}_{k}\right\|<0
$$

From the above discussion, we can find that the HIF can limit the noise interference to the H-infinity norm of the state estimation error within a restricted interference range. According to the first-order ECM, the input variable and state variable are defined as:

$$
\left\{\begin{array}{l}
u_{k}=I_{k} \\
x_{k}=\left[s_{k}, \quad V_{1, k}\right]^{T}
\end{array}\right.
$$

Then, the state space equation of system can be expressed as:

$$
\left\{\begin{array}{l}
F_{k}=A_{k} x_{k}+B_{k} u_{k} \\
y_{k}=V_{o c, k}-V_{1, k}-R_{0} u_{k}
\end{array}\right.
$$

where

$$
A_{k}=\left[\begin{array}{cc}
1 & 0 \\
0 & e^{-\frac{\Delta t}{R_{1} C_{1}}}
\end{array}\right], B_{k}=\left[\frac{\eta \Delta t}{Q_{n}}, \quad\left(1-e^{-\frac{\Delta t}{R_{1} C_{1}}}\right) R_{1, k}\right]^{T}
$$

Similar to the AEKF algorithm [21], the adaptive covariance calculation algorithm is added to the traditional HIF algorithm for updating the noise covariance matrix in the iterative process. Now, the general process of AHIF is illustrated in Table II. 
From the implementation process of FF-RLS and AHIF, we can find that all the remaining parameters of the state space model, except the battery capacity $Q_{n}$, can be updated periodically. To attain estimation of $Q_{n}$, in this study, the support vector machine (SVM) is employed.

Table II Specification of the test battery

\begin{tabular}{ll}
\hline Initialization & $\hat{x}_{0}^{+}=E\left(x_{0}\right), P_{0}^{+}=E\left[\left(x_{0}-\hat{x}_{0}^{+}\right)\left(x_{0}-\hat{x}_{0}^{+}\right)^{T}\right]$ \\
Prior estimate of state & $\hat{x}_{k}^{-}=F_{k-1} \hat{x}_{k-1}^{+}$ \\
Prior estimate of error covariance & $P_{k}^{-}=F_{k-1} P_{k-1}^{+} F_{k-1}^{T}+Q_{k-1}$ \\
Symmetric positive definite matrices & $\bar{S}_{k}=L_{k}^{T} S_{k} L_{k}$ \\
update & $\left(P_{k}^{-}\right)^{-1}-\theta \bar{S}_{k}+H_{k}^{T} R_{k}^{-1} H_{k}>0$ \\
Condition judgment & $e_{k}=y_{k}-H_{k} \hat{x}_{k}^{-}$ \\
Innovation update & $\hat{M}_{k}=\frac{1}{N} \sum_{i=k-N+1}^{k} e_{i} e_{i}^{T}, \hat{R}_{k}=\hat{M}_{k}-H_{k} P_{k}^{-} H_{k}^{T}$ \\
Adaptive estimation of measurement noise & $K_{k}=F_{k} P_{k}^{-}\left(I-\theta \bar{S}_{k} P_{k}^{-}+H_{k}^{T} R_{k}^{-1} H_{k} P_{k}^{-}\right)^{-1} H_{k}^{T} R_{k}^{-1}$ \\
matrix & $\hat{Q}_{k}=K_{k} \hat{M}_{k} K_{k}^{T}$ \\
Adain matrix update & $\hat{x}_{k}^{+}=\hat{x}_{k}^{-}+K_{k} e_{k}$ \\
Measurement update of state estimate & $P_{k}^{+}=P_{k}^{-}\left(I-\theta \bar{S}_{k} P_{k}^{-}+H_{k}^{T} R_{k}^{-1} H_{k} P_{k}^{-}\right)^{-1}$ \\
Measurement update of error covariance &
\end{tabular}

\section{B. The SOH Estimation Algorithm}

By comparing our proposed $\mathrm{SOH}$ estimation algorithm with other data driven algorithms, the SVM algorithm does not fall into the local extreme problem, and particularly it can be justified by rigorous mathematical proof. However, the general SVM shows complex solution process and intensive computation burden. The LS-SVM method is consequently developed to solve the nonlinear regression estimation problem with less computation load, and is proved suitable for battery SOH estimation [39-41]. The following equation is established, as:

$$
\min J(\boldsymbol{w}, \boldsymbol{\xi})=\frac{1}{2} \boldsymbol{w}^{T} \boldsymbol{w}+\frac{C}{2} \sum_{i=1}^{l} \boldsymbol{\xi}_{i}^{2}
$$

where $\xi=\left[\begin{array}{llll}\xi_{1} & \xi_{2} & \cdots & \xi_{l}\end{array}\right]$ is the deviation vector, and $C$ denotes the weight, which can be identified to find the optimal hyper plane. In addition, the following constraint need to be satisfied:

$$
y_{i}\left[\boldsymbol{w}^{T} \varphi\left(\mathbf{x}_{i}\right)+b\right]=1-\xi_{i}, i=1,2, \ldots, l
$$

The physical meaning of $\xi_{i}$ in (37) can be explained as follows. When the sample $\mathbf{x}_{i}$ lies outside the two critical hyperplanes, $\xi_{i}$ is less than zero and it indicates the negative distance from $\mathbf{x}_{i}$ to the nearest critical hyper plane. On the contrary, when $\mathbf{x}_{i}$ is located between two critical hyperplanes, $\xi_{i}$ is greater than zero; 
highlighting the positive distance from $\mathbf{x}_{i}$ to the nearest critical hyper plane. The Lagrange function, as shown in (38), is defined and the maximum condition of the function is solved to achieve the minimization.

The Lagrange equation is:

$$
L(\boldsymbol{w}, \boldsymbol{\beta}, \boldsymbol{\xi}, \boldsymbol{\alpha}, C)=J(\boldsymbol{w}, \boldsymbol{\xi})-\sum_{i=1}^{l} \boldsymbol{\alpha}_{i}\left(\boldsymbol{w}^{T} \cdot \Phi\left(\boldsymbol{x}_{i}\right)+b+\boldsymbol{\xi}_{i}-y_{i}\right)
$$

where $\boldsymbol{\alpha}_{i}$ represents the Lagrange multiplier. The optimization condition can be summarized as:

$$
\frac{\partial L}{\partial \boldsymbol{w}}=0, \frac{\partial L}{\partial \boldsymbol{\alpha}}=0, \frac{\partial L}{\partial \boldsymbol{b}}=0, \frac{\partial L}{\partial \xi}=0
$$

Given the above conditions, the following equations can be derived:

$$
\left\{\begin{array}{l}
\boldsymbol{w}=\sum_{i=1}^{l} \alpha_{i} \Phi\left(\boldsymbol{x}_{i}\right) \\
\boldsymbol{w} \cdot \Phi\left(\boldsymbol{x}_{i}\right)+b+\xi_{i}-y_{i}=0 \\
\sum_{i=1}^{l} \boldsymbol{\alpha}_{i}=0 \\
\boldsymbol{\alpha}_{i}=C \boldsymbol{\xi}_{i}
\end{array}\right.
$$

By solving (40), the solution can be obtained:

$$
f(x)=\sum_{i=1}^{l} \boldsymbol{\alpha}_{i} K\left(\boldsymbol{x}, \boldsymbol{x}_{i}\right)+b
$$

where $K\left(\boldsymbol{x}, \boldsymbol{x}_{i}\right)$ denotes the kernel function, and $K\left(\boldsymbol{x}, \boldsymbol{x}_{i}\right)=\Phi\left(\boldsymbol{x}_{i}\right)^{T} \Phi\left(\boldsymbol{x}_{i}\right)$. There are various types of kernel functions employed to solve the classification problem, such as Sigmoid kernel function, polynomial kernel function, radial basis kernel function (RBF), linear kernel function and Fourier kernel function [42]. Among these kernel functions, the RBF kernel function can map the sample nonlinearly to a higher dimensional space with less numerical burden. Given these advantages of RBF kernel function, we selected it as the kernel function in this study,

$$
K\left(\boldsymbol{x}, \boldsymbol{x}_{i}\right)=\exp \left(-\frac{\left\|\boldsymbol{x}-\boldsymbol{x}_{i}\right\|^{2}}{2 \gamma^{2}}\right)
$$

where $\gamma$ denotes the width of RBF. Actually, the selection of feature variables plays a critical role in improvement of learning performance. For $\mathrm{SOH}$ estimation, the selected characteristics reflect the capacity variation of different cycle numbers [43], and Ref. [44] pointed out that the charging time of CC stage decreases with degradation. In this study, the battery charging voltage profiles with different $\mathrm{SOH}$ levels are depicted in Fig. 2, and it can be observed that the CC stage obviously becomes shorter when the battery ages and the time 
interval of the equal charging voltage difference gradually decreases. Hence, an appropriate feature variable from the $\mathrm{CC}$ charging voltage can be extracted to estimate the battery $\mathrm{SOH}$. In this study, the charging duration $\left[\begin{array}{ll}V_{\text {start }} & V_{\text {stop }}\end{array}\right]$ is selected as the characteristic variable, where $V_{\text {start }}$ and $V_{\text {stop }}$ denote the starting and ending voltage value, respectively.

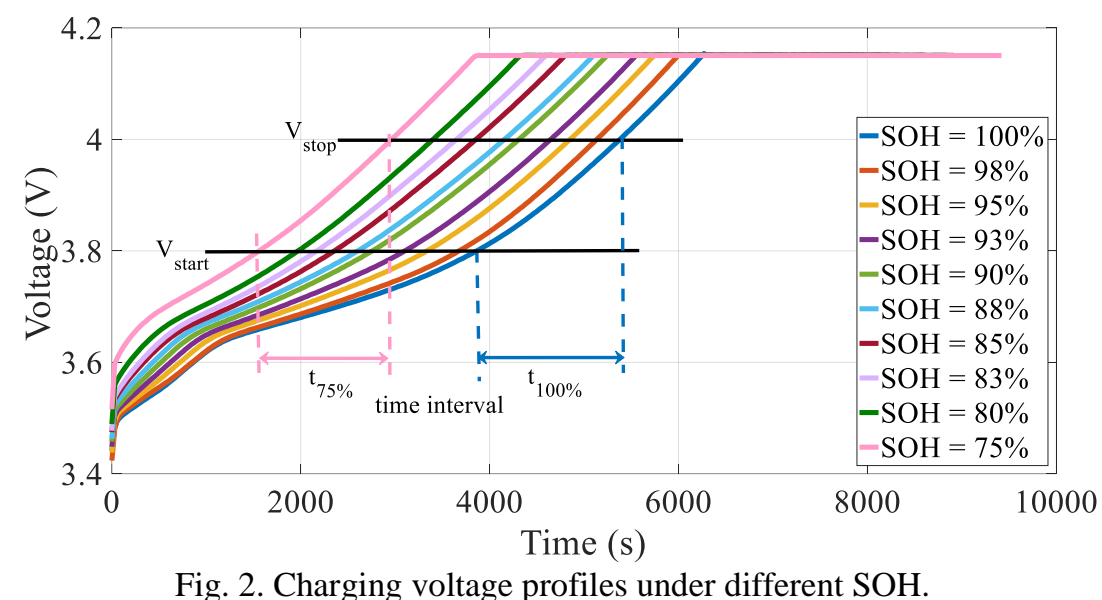

Fig. 2. Charging voltage profiles under different $\mathrm{SOH}$.

\section{Fusion Algorithm}

On the basis of $\mathrm{SOH}$ estimation, the adaptive fusion algorithm based on AHIF is therefore constructed to achieve the SOC estimation, as shown in Fig. 3. As can be seen, the fusion algorithm is divided into five parts: measurement, decision, parameters identification, $\mathrm{SOH}$ estimation and SOC estimation. When the battery operates, the measurement module monitors and records the battery current and terminal voltage. Then, the battery parameters are identified by the parameter identification module according to the measurement. When the decision module determines that the battery is in the $\mathrm{CC}$ charging state, the $\mathrm{SOH}$ estimation module is activated to estimate the battery $\mathrm{SOH}$, thus adaptively updating the battery capacity value. Finally, the SOC estimation module conducts the estimation by the AHIF algorithm with the updated model parameters and capacity. 


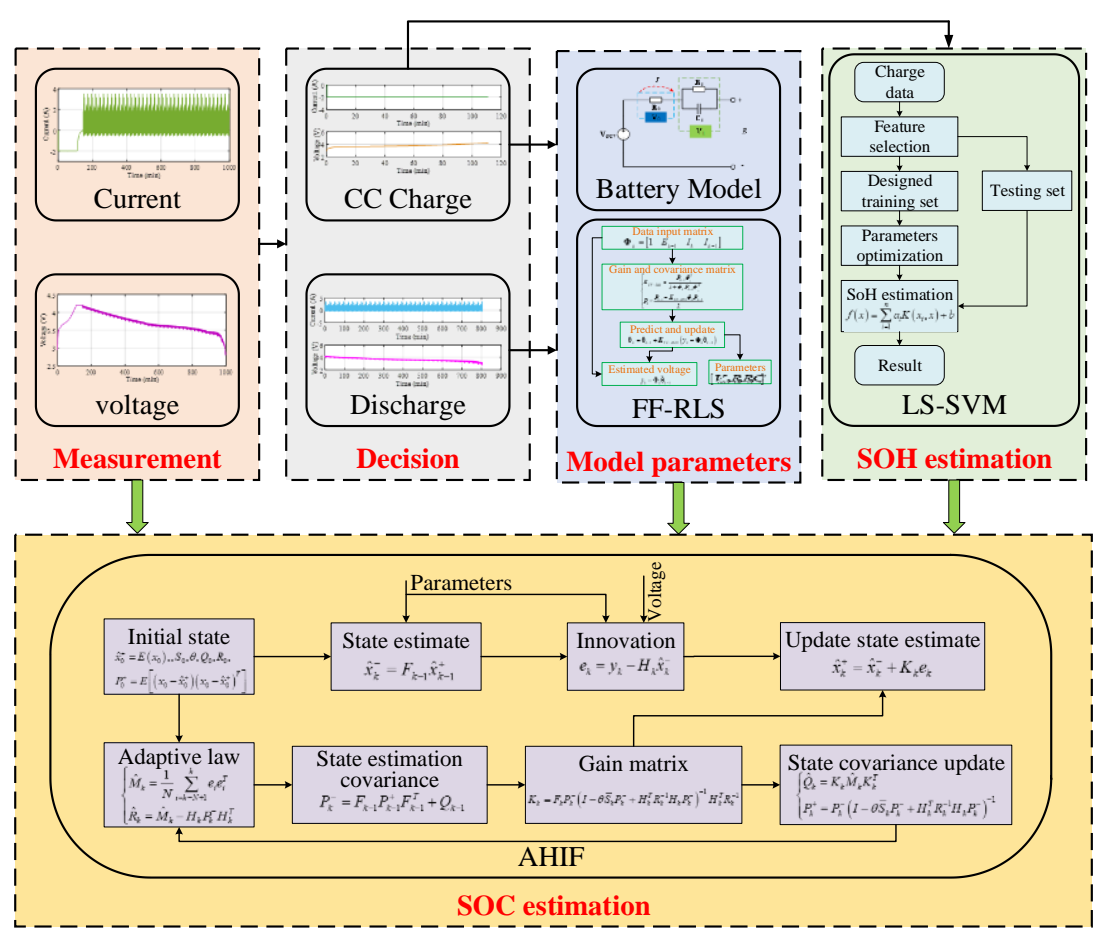

Fig. 3. Flowchart of the adaptive fusion algorithm.

According to the above estimation procedures, the model parameters, including ohmic resistance, polarization resistance, polarization capacity, OCV and battery capacity, can be timely updated. Thus, we can say that the battery SOC can be accurately estimated all the time, independent on the battery operating conditions. The detailed validation and discussion will be illustrated in the next section.

\section{EXPERIMENTAL VALIDATION AND DISCUSSION}

To estimate the SOC, a preliminary task is that the algorithm needs to know the battery capacity value. Hence, in this section, the SOH estimation and validation is conducted first, followed by the SOC estimation.

\section{A. SOH Validation}

First, a series of experiments are conducted to acquire enough data for training and validating the LS-SVM model. During the aging test, a constant current-constant voltage (CC-CV) charging strategy with the current of $0.5 \mathrm{C}$ is implemented in the charging process. The cells are discharged with the current of $1 \mathrm{C}$, and the cut-off current of $\mathrm{CV}$ stage is $0.02 \mathrm{C}$. The whole test dataset is divided into three segments, wherein the dataset of cell 1 is divided into two parts with one for training and the other for verification; and the dataset of cell 2 is used to validate the model. According to the estimation algorithm of SOH addressed previously, the feature voltage range is set from $3.58 \mathrm{~V}$ to $4.15 \mathrm{~V}$. The $\mathrm{SOH}$ estimation results, the referred values and their difference are 
depicted in Fig. 4 and the comparison criteria, including the mean absolute error, maximum absolute error, mean square error (MSE) and RMSE, are quantified in Table III. It can be found that the mean absolute error, maximum absolute error, MSE and RMSE are respectively $0.37 \%, 1.86 \%, 2.28 \times 10^{-5}$ and $0.48 \%$, justifying the feasibility of proposed algorithm. In addition, a raw dataset of another cell, namely cell 2 , is applied to validate the performance of the training model. Fig. 4 (c) and (d) depict the estimation results. Although the estimation error of cell 2 is higher than the calibrated experiments, most of the maximum absolute error are still less than $2 \%$ (except several isolated points), highlighting the preferable performance of proposed estimation algorithm for $\mathrm{SOH}$.

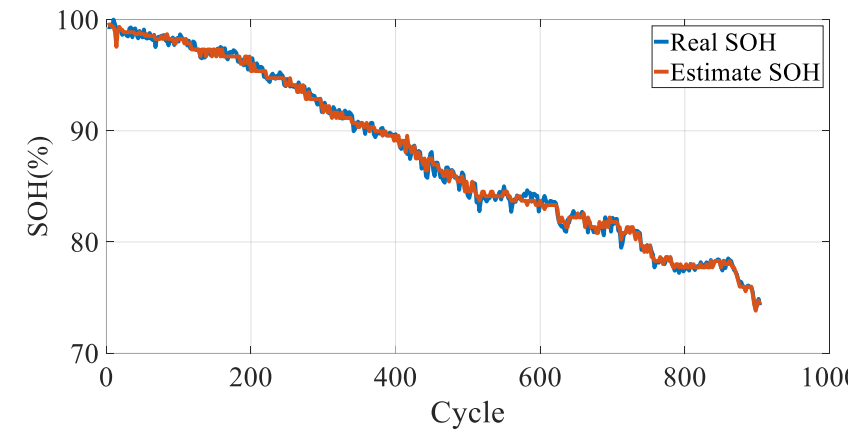

(a)

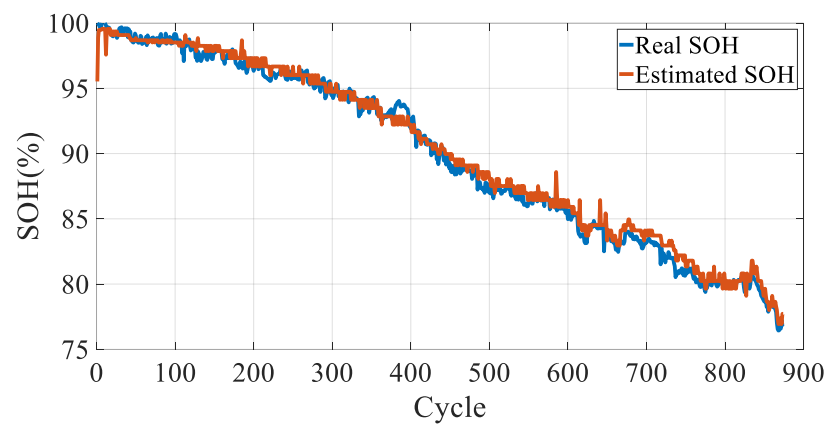

(c)

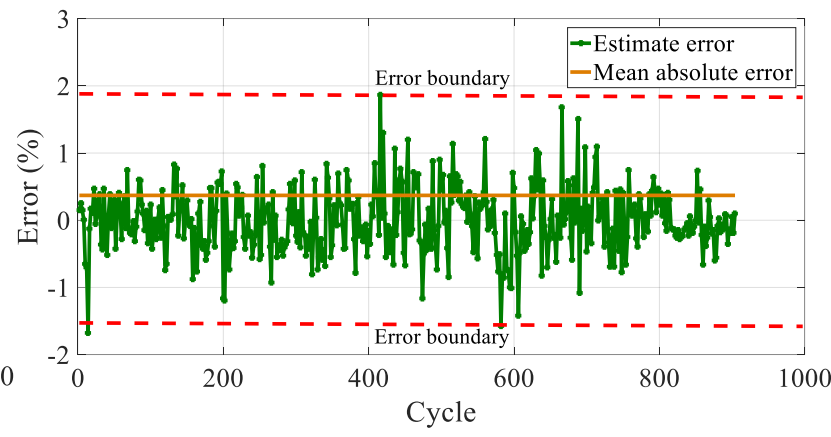

(b)

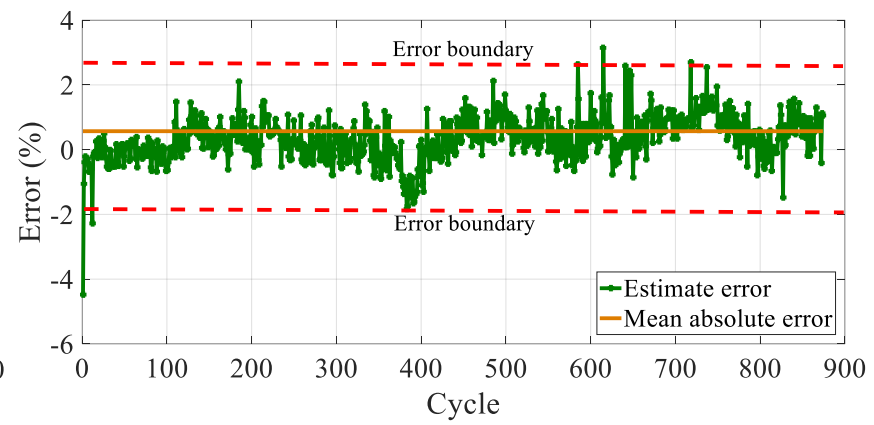

(d)

Fig. 4. SOH estimation results of two cells. (a) Measured and estimated SOH for cell 1; (b) Estimation error for cell 1; (c) Measured and estimated SOH for cell 2; (d) Estimation error for cell 2.

Table III. Estimation results of multiple battery experiments.

\begin{tabular}{ccccc}
\hline Cell number & $\begin{array}{c}\text { Mean absolute } \\
\text { error }(\%)\end{array}$ & $\begin{array}{c}\text { Maximum } \\
\text { absolute error }(\%)\end{array}$ & MSE & RMSE (\%) \\
\hline Cell 1 & 0.37 & 1.86 & $2.28 \mathrm{e}^{-05}$ & 0.48 \\
Cell 2 & 0.58 & 4.03 & $5.61 \mathrm{e}^{-05}$ & 0.75 \\
\hline
\end{tabular}

\section{B. Comparison of SOC Estimation with Different Algorithms}

To evaluate the SOC estimation performance, three commonly used methods, including EKF method, AEKF and HIF, are employed. It is necessary to note that all the battery parameters employed to estimate the SOC are identified based on the improved FF-RLS algorithm. The current profiles acquired based on urban 
dynamometer driving schedule (UDDS) is repetitively implemented until the terminal voltage of battery drops to the cut-off voltage, i.e., $2.75 \mathrm{~V}$. The reference initial SOC value is $100 \%$, and to validate the independence of different methods on the initial value, the initial SOC when applying the algorithm is set to $60 \%$. The detailed comparison of different algorithms is illustrated in Fig. 5 and Table IV. It can be clearly found that EKF, HIF, AEKF and proposed algorithm can all compensate the large error incurred by the preset initial differences. As listed in Table IV, the duration to reach the reference SOC value, which defined as the time when the estimate is stabilized within the 5\% error bound, is respectively $45 \mathrm{~s}, 40 \mathrm{~s}, 46 \mathrm{~s}$ and $30 \mathrm{~s}$, highlighting that the proposed algorithm responses faster than other algorithms. It can also be observed that the SOC estimation based on AHIF attains least estimation error during the whole discharging process, and the maximum absolute error, mean absolute error and RMSE are $0.7 \%, 0.42 \%$, and $0.6 \%$, respectively. Obviously, the AHIF exhibits highest estimation accuracy among these four filters. The reason is that the AHIF does not assume that the statistical characteristics of noise are known in advance, that is imperative in Kalman filter [3], and instead suppresses the norm of interference into the designated range, thereby enabling the observer to solve the bounded signal and improving its robustness dramatically.

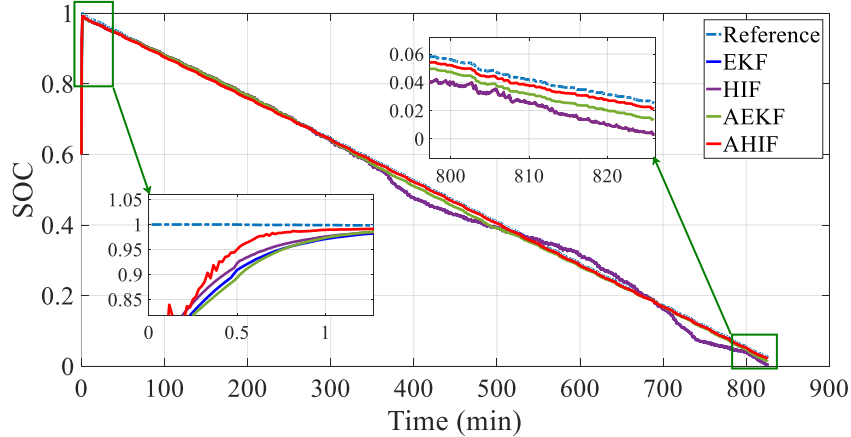

(a)

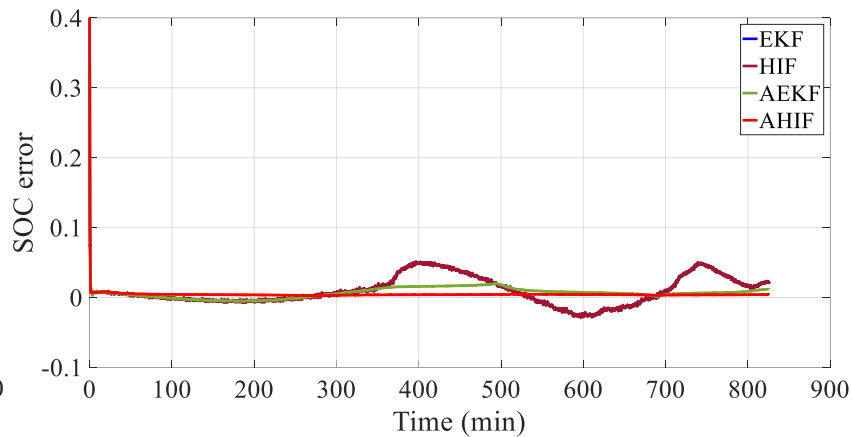

(b)

Fig. 5. Results of SOC estimation in case of different estimators: (a) referenced and estimated SOC and (b) corresponding error.

Table IV. Comparison results with different algorithms.

\begin{tabular}{ccccc}
\hline Algorithm & $\begin{array}{c}\text { Convergence } \\
\text { time }(\mathrm{s})\end{array}$ & $\begin{array}{c}\text { Maximum absolute } \\
\text { error }(\%)\end{array}$ & $\begin{array}{c}\text { Mean absolute } \\
\text { error }(\%)\end{array}$ & RMSE \\
\hline EKF & 45 & 5.08 & 1.6 & 2.22 \\
HIF & 40 & 5.144 & 1.6 & 2.21 \\
AEKF & 46 & 2.014 & 0.79 & 1.05 \\
AHIF & 30 & 0.7 & 0.42 & 0.6 \\
\hline
\end{tabular}




\section{SOC Estimation at Different Temperatures}

All the above validations and comparisons are conducted at room temperature. It is vital to verify the thermal adaptability of the algorithm existing in practice. As described in Section II, the battery parameters change dramatically with operating temperature. Here, the performance of designed SOC estimation algorithm is further investigated under different operating conditions, such as constant low temperature $\left(-20^{\circ} \mathrm{C}\right)$ and timevarying temperature. In this discussion, the effects of inaccurate initial SOC is also considered to evaluate the convergence performance of proposed algorithm at different temperatures. The initial value is set to $85 \%$ when estimating the SOC, and obviously, the initial error is $15 \%$.

\section{1). Evaluation Results at Low Temperature}

It is well acknowledged that battery capacity and power output can degrade at low temperature. This may affect battery SOC estimation accuracy to large extent. Similar as the experiment before, the UDDS current profile is still imposed at $-20^{\circ} \mathrm{C}$. Note that the cell is placed in the thermal controlled chamber for 3 hours until the experiment starts. Fig. 6 demonstrates the estimated SOC, estimated voltage and corresponding errors when compared with their reference values. The SOC estimation result and corresponding error are provided in Fig. 6 (a) and (b), respectively; in which we can find that the overall error is less than $1 \%$ except the initially arbitrary setting difference. In addition, it is obviously found that the output voltage of model tracks the measured value precisely, contributing to accurate estimation of SOC, and the mean absolute error is less than $0.014 \mathrm{~V}$, only accounting for $0.38 \%$ of the nominal voltage. All the statistical comparisons at different temperatures are summarized in Table V. In summary, the verification test proves that the proposed method can accurately estimate the SOC in a low temperature condition.

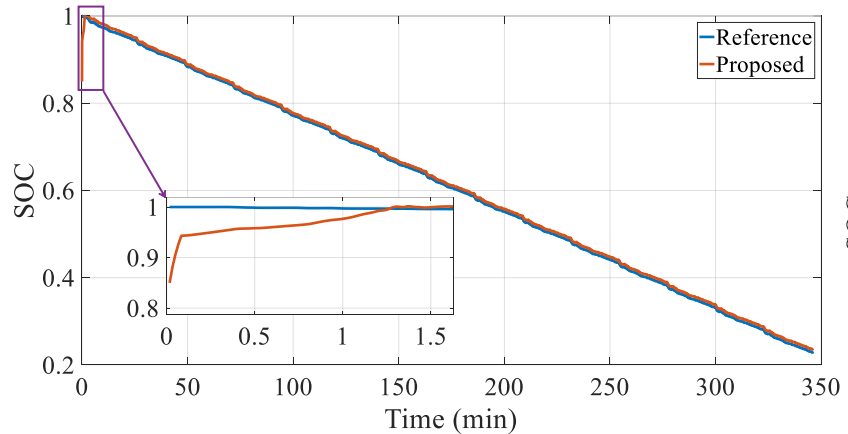

(a)

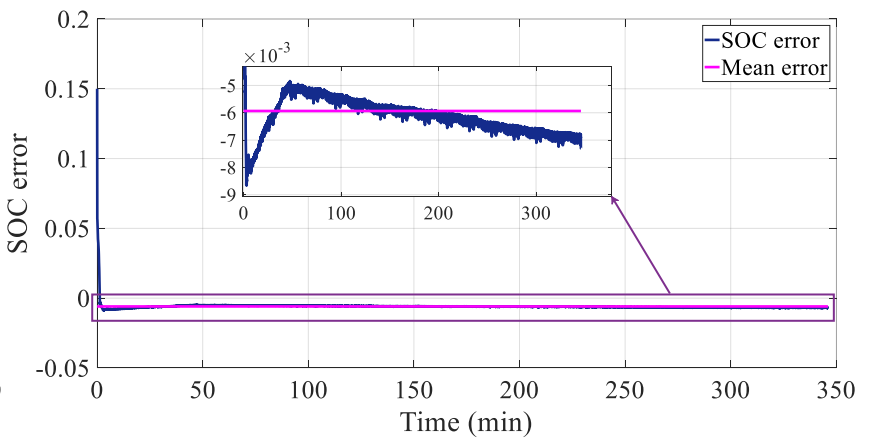

(b) 


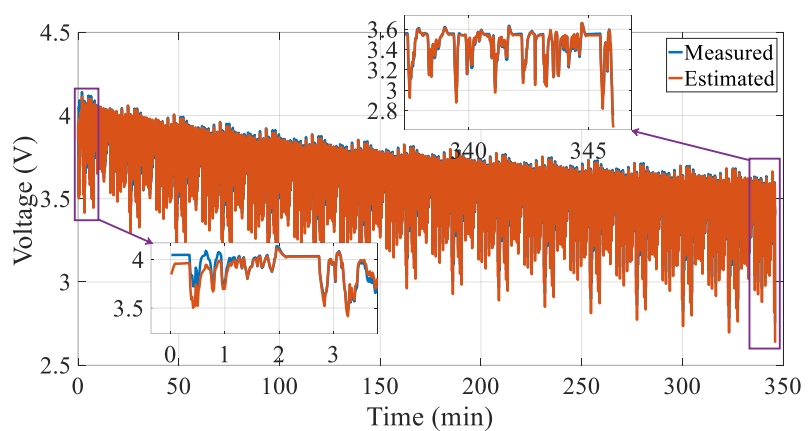

(c)

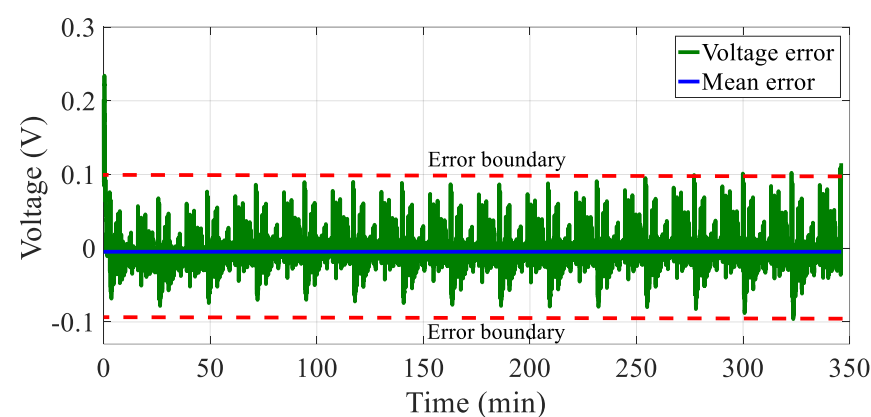

(d)

Fig. 6. The SOC validation results in case of $-20{ }^{\circ} \mathrm{C}$ : (a) the comparison results of the reference SOC and estimated SOC; (b) SOC estimation error; (c) the comparison results of the measured voltage and estimated voltage; (d) voltage estimation error.

\section{2). Evaluation Results with Time-Varying Temperature}

In practice, batteries are not easy to operate with constant temperature. To further validate the performance of proposed algorithm, the operating temperature continuously varies from $30{ }^{\circ} \mathrm{C}$ to $5{ }^{\circ} \mathrm{C}$. Likewise, the cell is placed in the thermal controlled chamber at $30{ }^{\circ} \mathrm{C}$ for three hours. Fig. 7 (a) depicts the temperature variation. The SOC estimation result and error are shown in Fig. 7 (b) and (c), where the GA-AHIF indicates the model parameters are identified by GA, and the SOC is estimated by AHIF. It can be seen from Fig. 7 (b), both the GA-AHIF and proposed SOC estimation method can quickly offset the initial error and track the reference curve precisely. The convergence time, maximum absolute error, mean absolute error and RMSE of the proposed algorithm are $27 \mathrm{~s}, 0.79 \%, 0.24 \%$ and $0.42 \%$, respectively. However, the convergence time of GA-AHIF based method is around four times longer than the proposed method. Besides, the GA-AHIF method incurs larger estimation error, which gradually increases to $2.68 \%$ in the end of the test. Fig. 7 (d) and (e) present the measured voltage, model output and errors. Similar as the SOC, the terminal voltage based on the proposed method is much smoother and more accurate than that of the GA-AHIF method, manifesting its strong adaptability to temperature variation. Thanks to the improved FF-RLS algorithm, the model parameters can be accurately adjusted online even in the case of low-current excitation. The online identified OCV is shown in Fig. 7 (f). It is obvious that the OCV changes with the temperature, and in other words, the necessity of updating the OCV dynamically according to temperature variation is justified. 


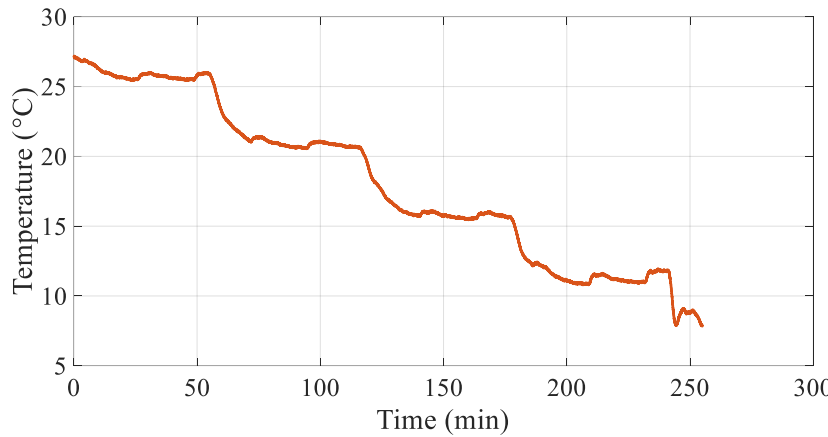

(a)

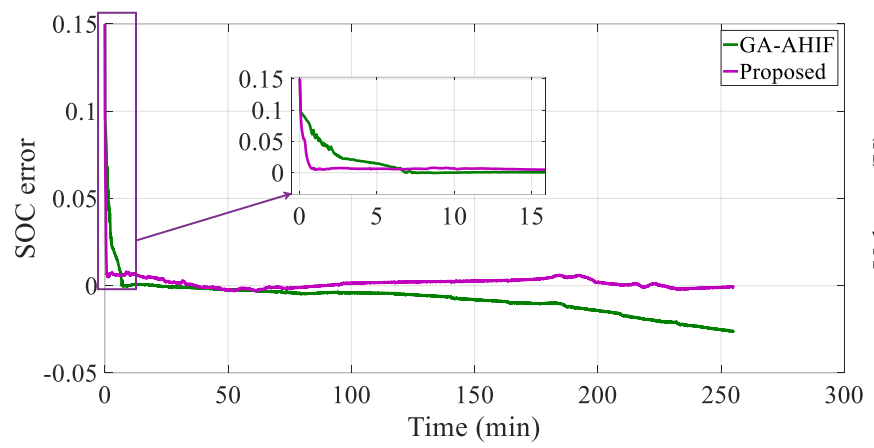

(c)

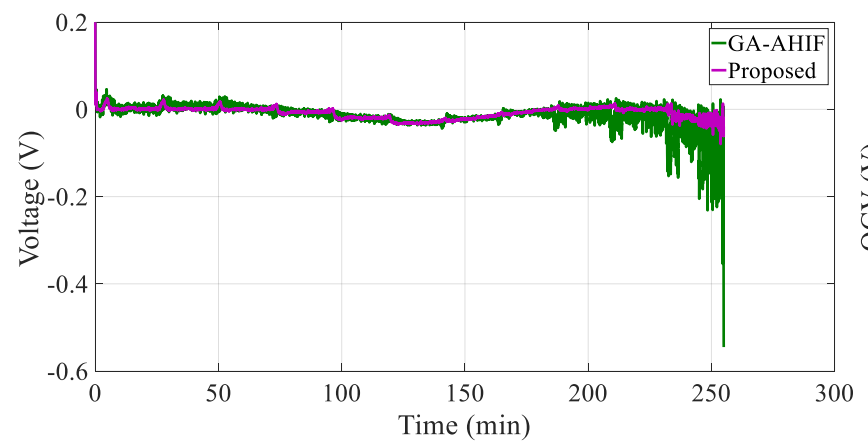

(e)

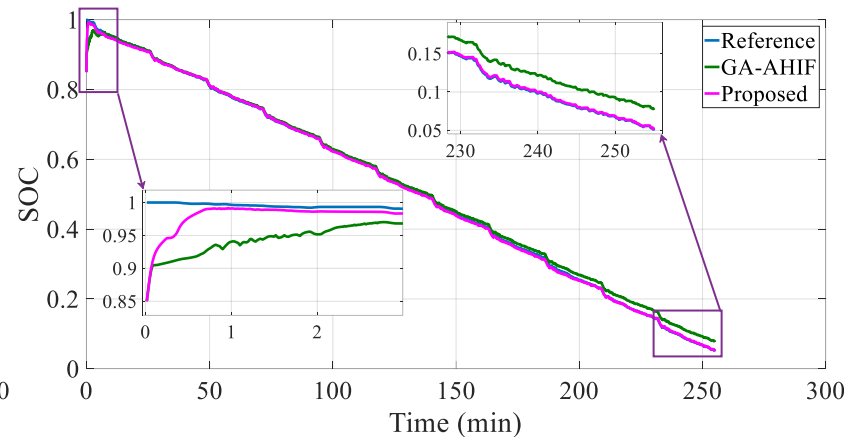

(b)

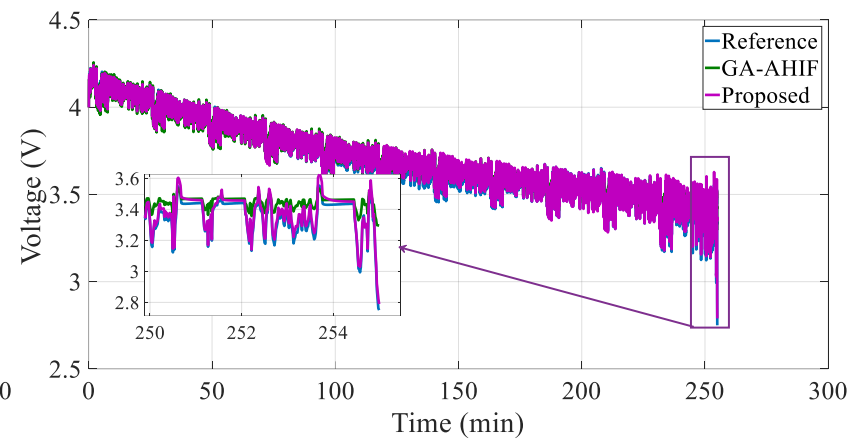

(d)

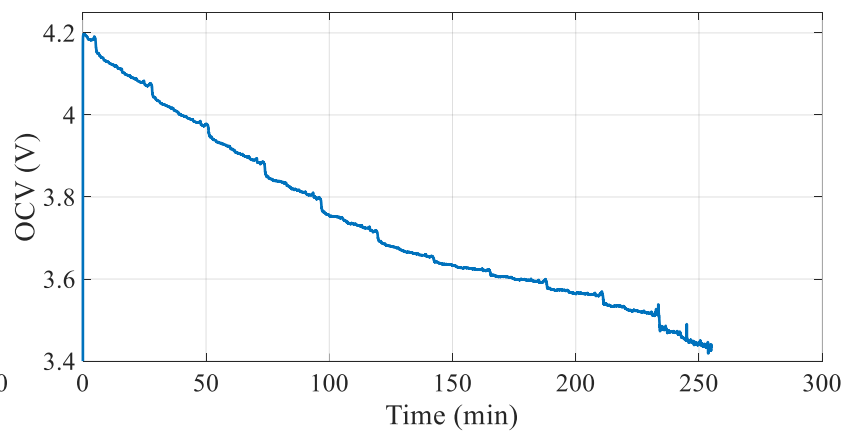

(f)

Fig. 7. Results of SOC estimation in case of time-varying temperature: (a) Temperature versus time; (b) SOC versus time; (c) SOC estimation error versus time; (d) OCV versus time; (e) Voltage estimation comparison result versus time; (f) Voltage estimation error versus time.

Table V Statistical data of estimated SOC and voltage at different temperatures

\begin{tabular}{lllllllll}
\hline & & SOC & \multicolumn{5}{c}{ Voltage } \\
\cline { 3 - 9 } Temperature & Method & $\begin{array}{l}\text { Convergence } \\
\text { time }(\mathrm{s})\end{array}$ & $\begin{array}{l}\text { Maximum } \\
\text { absolute } \\
\text { error }(\%)\end{array}$ & $\begin{array}{l}\text { Mean } \\
\text { absolute } \\
\text { error }(\%)\end{array}$ & $\begin{array}{l}\text { RMSE } \\
(\%)\end{array}$ & $\begin{array}{l}\text { Maximum } \\
\text { absolute } \\
\text { error }(\mathrm{V})\end{array}$ & $\begin{array}{l}\text { Mean } \\
\text { absolute } \\
\text { error }(\mathrm{V})\end{array}$ & $\begin{array}{l}\text { RMSE } \\
(\mathrm{V})\end{array}$ \\
\hline Constant & Proposed & 36 & 0.8 & 0.62 & 0.67 & 0.115 & 0.0132 & 0.0198 \\
Variable & Proposed & 27 & 0.79 & 0.24 & 0.42 & 0.0795 & 0.0103 & 0.0148 \\
& GA-AHIF & 129 & 2.68 & 0.93 & 1.3 & 0.545 & 0.0169 & 0.0284 \\
\hline
\end{tabular}

\section{SOC Estimation with Aged Cells}

Based on the proposed fusion estimation method, cells with different aging status are tested to validate the performance of proposed SOC estimation algorithm. The battery is cycled with UDDS current at room temperature. By extracting the characteristic parameters of the charging process, the battery SOH is firstly estimated. When the battery is discharged, the updated capacity is imported to estimate the SOC. In this case, 
the initial SOC is set to $20 \%$ for all the aging cells, and obviously the difference is $80 \%$. The SOC estimation results in terms of aging cells are illustrated in Fig. 8 and Table VI, respectively. As can be seen, with the aging of battery, the total discharging time decreases gradually in the same working condition. The estimated SOC against different aging status can all converge to the referred values, proving the robustness and stability of proposed algorithm when dealing with aged cells. The maximum estimation error of SOC with respect to four different SOH status locates within the same region which corresponds to the SOC range from $60 \%$ to $30 \%$. This is because the SOC-OCV correlation is quite flat in this range, as depicted in Fig. 8 (c). The SOC estimation error based on the proposed method can be restricted within $1.2 \%$ under this case. The remaining battery parameters including OCV, ohmic resistance, polarization resistance and time constant are shown in Figs. 8 (c) to (f). We can find that only slight difference among different OCV curves exists when the battery ages. When SOC drops to $4 \%$ from $60 \%$, the OCV decreases by $0.016 \mathrm{~V}$, which may lead to the SOC estimation error of 1.4\%. Nonetheless, the battery parameters change obviously under different aging conditions. Hence, we can conclude that real-time update of battery parameters under different aging status are imperative to improve the accuracy of SOC estimation. The comparison results clearly prove the effectiveness of proposed SOC estimation algorithm in a wide life cycle.

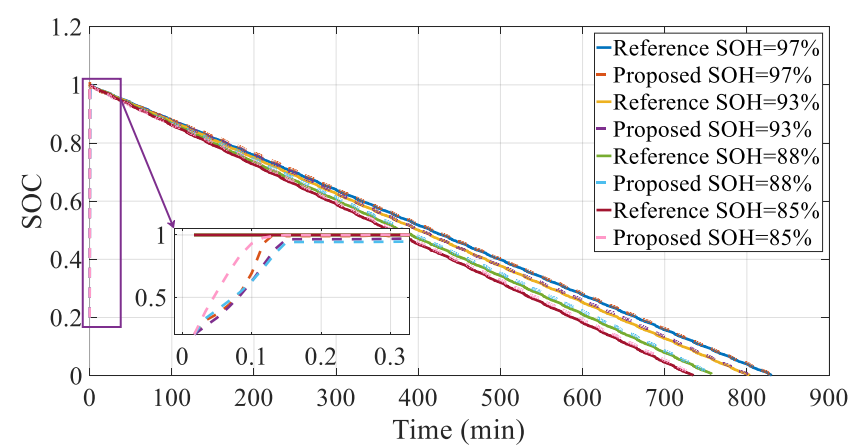

(a)

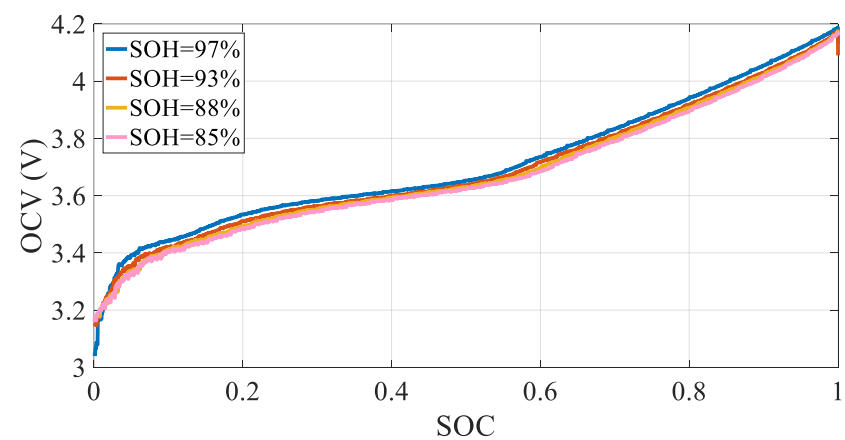

(c)

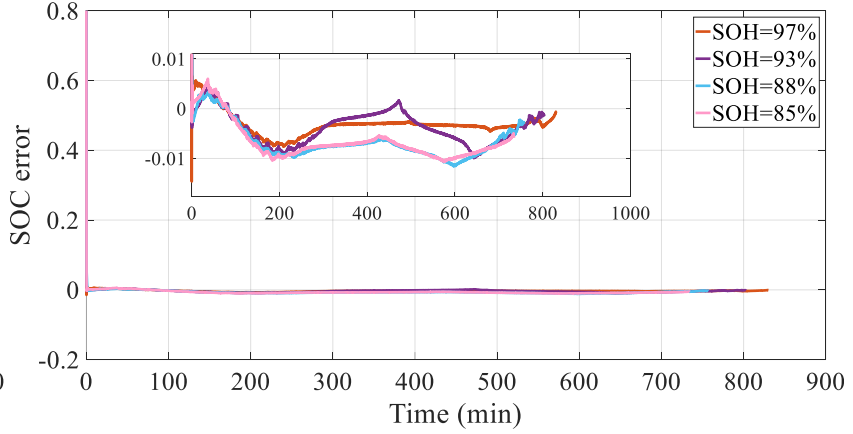

(b)

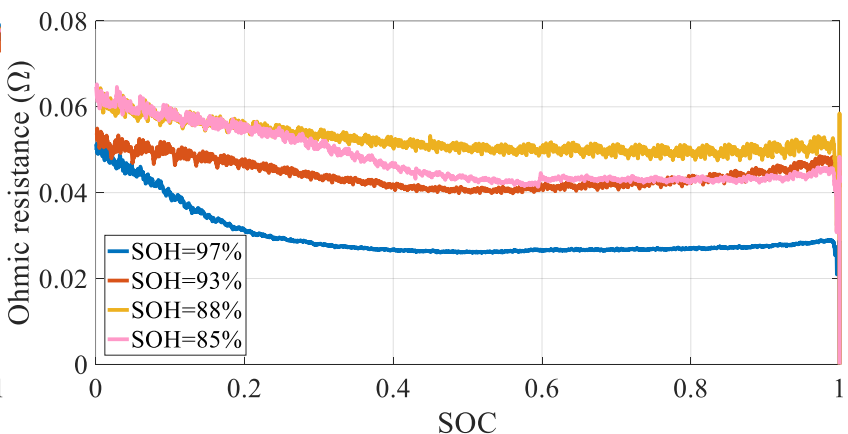

(d) 


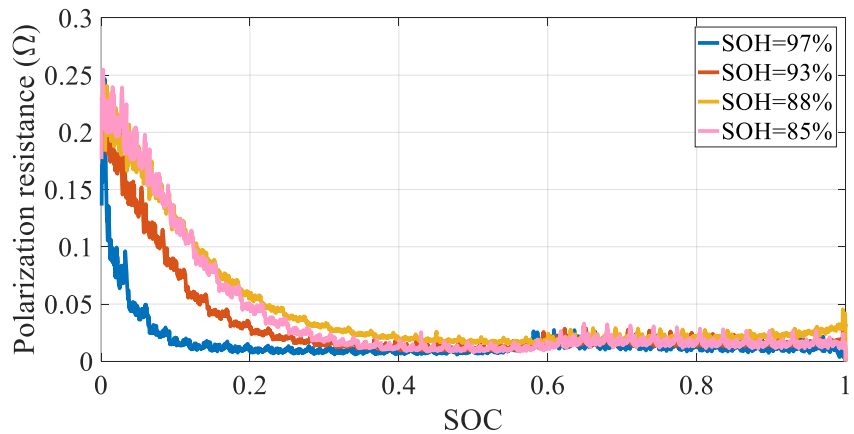

(e)

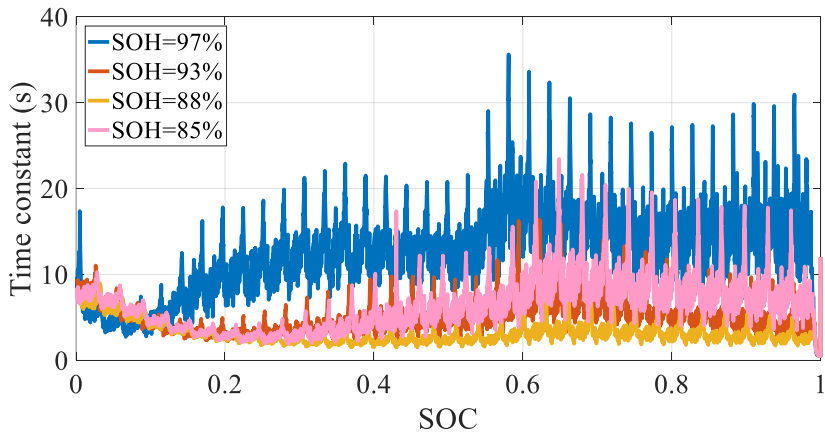

(f)

Fig. 8. Results of SOC estimation in case of aging cells: (a) reference and estimated SOC and (b) SOC error; (c) estimated OCV; (d) estimated ohmic resistance; (e) estimated polarization resistance; (f) estimated time constant.

Table VI Summarization of SOC and voltage estimation results with aging cell

\begin{tabular}{|c|c|c|c|c|c|c|c|}
\hline \multirow[b]{2}{*}{$\mathrm{SOH}$} & \multicolumn{4}{|l|}{$\mathrm{SOC}$} & \multicolumn{3}{|l|}{ Voltage } \\
\hline & $\begin{array}{l}\text { Convergence } \\
\text { time (s) }\end{array}$ & $\begin{array}{l}\text { Maximum } \\
\text { absolute } \\
\text { error }(\%)\end{array}$ & $\begin{array}{l}\text { Mean } \\
\text { absolute } \\
\text { error }(\%)\end{array}$ & $\begin{array}{l}\text { RMSE } \\
(\%)\end{array}$ & $\begin{array}{l}\text { Maximum } \\
\text { absolute } \\
\text { error (V) }\end{array}$ & $\begin{array}{l}\text { Mean } \\
\text { absolute } \\
\text { error (V) }\end{array}$ & $\begin{array}{l}\text { RMSE } \\
\text { (V) }\end{array}$ \\
\hline $97 \%$ & 33 & 0.73 & 0.37 & 0.76 & 0.052 & 0.0082 & 0.013 \\
\hline $93 \%$ & 36 & 0.97 & 0.43 & 0.86 & 0.059 & 0.0082 & 0.0128 \\
\hline $88 \%$ & 57 & 1.16 & 0.7 & 1.02 & 0.067 & 0.0092 & 0.0142 \\
\hline $85 \%$ & 47 & 1.03 & 0.71 & 0.93 & 0.088 & 0.0094 & 0.0146 \\
\hline
\end{tabular}

\section{CONCLUSION}

In this paper, an adaptive fusion algorithm is proposed to investigate the influence of battery degradation and dynamic working temperature on the state of charge estimation. An improved online identification algorithm based on the improved recursive least square method with the forgetting factor is applied to identify model parameters over a wide temperature range of $-20{ }^{\circ} \mathrm{C}$ to $50{ }^{\circ} \mathrm{C}$. The least square support vector machine algorithm is employed to conduct the accurate state of health estimation, which in turn assists estimation of battery state of charge. The experimental results reveal that the state of health estimation error is less than $2 \%$. The adaptive H-infinity filter is proposed to estimate the state of charge based on the dynamically updated state of health and precise battery model. By comparing with the commonly used extended Kalman filter, adaptive extended Kalman filter and H-infinity filter algorithms, the proposed algorithm exhibits higher estimation accuracy, fast convergence speed, and better adaptation to variation of external operating temperatures and battery degradation. The comprehensive evaluation of algorithm highlights its broad application potential in estimation of state of charge and state of health for lithium-ion batteries.

Next step work will be focused on the systematical performance evaluation when applying the algorithm in a battery pack. In particular, when inconsistency of temperature and capacity exists among cells in the pack, how to deal with the imbalance and supply the authoritative estimation needs to be further investigated. 


\section{ACKNOWLEDGEMENTS}

This work was supported in part by the National Natural Science Foundation (No. 51775063 and No. 61763021), in part by the National Key R\&D Program of China (No. 2018YFB0104000), in part by the Fundamental Research Funds for the Central Universities (No. 2018CDQYQC0035), and in part by the EUfunded Marie Skłodowska-Curie Individual Fellowships Project under Grant 845102-HOEMEV-H2020MSCA-IF-2018.

\section{REFERENCES}

[1] C. Zhang, F. Yang, X. Ke, Z. Liu, and C. Yuan, "Predictive modeling of energy consumption and greenhouse gas emissions from autonomous electric vehicle operations," Applied Energy, vol. 254, p. 113597, 2019.

[2] Y. Liu, J. Li, Z. Chen, D. Qin, and Y. Zhang, "Research on a multi-objective hierarchical prediction energy management strategy for range extended fuel cell vehicles," Journal of Power Sources, vol. 429, pp. 55-66, 2019.

[3] P. Shrivastava, T.K. Soon, M.Y.I.B. Idris, and S. Mekhilef, "Overview of model-based online state-of-charge estimation using Kalman filter family for lithium-ion batteries," Renewable and Sustainable Energy Reviews, vol. 113, p. 109233, 2019.

[4] X. Li, L. Zhang, Z. Wang, and P. Dong, "Remaining useful life prediction for lithium-ion batteries based on a hybrid model combining the long short-term memory and Elman neural networks," Journal of Energy Storage, vol. 21, pp. 510-518, 2019.

[5] K. Liu, K. Li, Q. Peng, and C. Zhang, "A brief review on key technologies in the battery management system of electric vehicles," Frontiers of Mechanical Engineering, vol. 14, pp. 47-64, 2019.

[6] H. Rahimi-Eichi, U. Ojha, F. Baronti, and M. Chow, "Battery Management System: An Overview of Its Application in the Smart Grid and Electric Vehicles," IEEE Industrial Electronics Magazine, vol. 7, pp. 4-16, 2013.

[7] C. Chen, R. Xiong, R. Yang, W. Shen, and F. Sun, "State-of-charge estimation of lithium-ion battery using an improved neural network model and extended Kalman filter," Journal of Cleaner Production, vol. 234, pp. 11531164, 2019.

[8] X. Li, Z. Wang, and J. Yan, "Prognostic health condition for lithium battery using the partial incremental capacity and Gaussian process regression," Journal of Power Sources, vol. 421, pp. 56-67, 2019.

[9] Z. Chen, Q. Xue, R. Xiao, Y. Liu, and J. Shen, "State of Health Estimation for Lithium-ion Batteries Based on Fusion of Autoregressive Moving Average Model and Elman Neural Network," IEEE Access, vol. 7, pp. 102662102678, 2019.

[10] T. Feng, L. Yang, X. Zhao, H. Zhang, and J. Qiang, "Online identification of lithium-ion battery parameters based on an improved equivalent-circuit model and its implementation on battery state-of-power prediction," Journal of Power Sources, vol. 281, pp. 192-203, 2015.

[11] X. Li, Z. Wang, L. Zhang, C. Zou, and D.D. Dorrell, "State-of-health estimation for Li-ion batteries by combing the incremental capacity analysis method with grey relational analysis," Journal of Power Sources, vol. 410-411, pp. 106-114, 2019. 
[12] F. Guo, G. Hu, and R. Hong, "A parameter adaptive method with dead zone for state of charge and parameter estimation of lithium-ion batteries," Journal of Power Sources, vol. 402, pp. 174-182, 2018.

[13] S.-C. Huang, K.-H. Tseng, J.-W. Liang, C.-L. Chang, and G.M. Pecht, "An Online SOC and SOH Estimation Model for Lithium-Ion Batteries," Energies, vol. 10, 2017.

[14] F. Yang, X. Song, F. Xu, and K. Tsui, "State-of-Charge Estimation of Lithium-Ion Batteries via Long Short-Term Memory Network," IEEE Access, vol. 7, pp. 53792-53799, 2019.

[15] S. Nejad, D.T. Gladwin, and D.A. Stone, "A systematic review of lumped-parameter equivalent circuit models for real-time estimation of lithium-ion battery states," Journal of Power Sources, vol. 316, pp. 183-196, 2016.

[16] X. Lai, Y. Zheng, and T. Sun, "A comparative study of different equivalent circuit models for estimating state-ofcharge of lithium-ion batteries," Electrochimica Acta, vol. 259, pp. 566-577, 2018.

[17] R. Xiong, L. Li, Z. Li, Q. Yu, and H. Mu, "An electrochemical model based degradation state identification method of Lithium-ion battery for all-climate electric vehicles application," Applied Energy, vol. 219, pp. 264-275, 2018.

[18] A. Jokar, B. Rajabloo, M. Désilets, and M. Lacroix, "Review of simplified Pseudo-two-Dimensional models of lithium-ion batteries," Journal of Power Sources, vol. 327, pp. 44-55, 2016.

[19] M. Ye, H. Guo, and B. Cao, "A model-based adaptive state of charge estimator for a lithium-ion battery using an improved adaptive particle filter," Applied Energy, vol. 190, pp. 740-748, 2017.

[20] G.L. Plett, "Extended Kalman filtering for battery management systems of LiPB-based HEV battery packs: Part 3. State and parameter estimation," Journal of Power Sources, vol. 134, pp. 277-292, 2004.

[21] Z. Chen, X. Li, J. Shen, W. Yan, and R. Xiao, "A Novel State of Charge Estimation Algorithm for Lithium-Ion Battery Packs of Electric Vehicles," Energies, vol. 9, 2016.

[22] J. Peng, J. Luo, H. He, and B. Lu, "An improved state of charge estimation method based on cubature Kalman filter for lithium-ion batteries," Applied Energy, vol. 253, p. 113520, 2019.

[23] C. Huang, Z. Wang, Z. Zhao, L. Wang, C.S. Lai, and D. Wang, "Robustness Evaluation of Extended and Unscented Kalman Filter for Battery State of Charge Estimation," IEEE Access, vol. 6, pp. 27617-27628, 2018.

[24] M. Gholizadeh and A. Yazdizadeh. (2019, State of charge estimation of a lithium-ion battery using robust nonlinear observer approach. IET Electrical Systems in Transportation 9(1), 1-7. Available: https://digitallibrary.theiet.org/content/journals/10.1049/iet-est.2018.0002

[25] C. Chen, R. Xiong, and W. Shen, "A lithium-ion battery-in-the-loop approach to test and validate multiscale dual $\mathrm{H}$ infinity filters for state-of-charge and capacity estimation," IEEE Transactions on power Electronics, vol. 33, pp. 332-342, 2017.

[26] Z. Wei, J. Zhao, C. Zou, T.M. Lim, and K.J. Tseng, "Comparative study of methods for integrated model identification and state of charge estimation of lithium-ion battery," Journal of Power Sources, vol. 402, pp. 189197, 2018

[27] S. Peng, X. Zhu, Y. Xing, H. Shi, X. Cai, and M. Pecht, "An adaptive state of charge estimation approach for lithium-ion series-connected battery system," Journal of Power Sources, vol. 392, pp. 48-59, 2018.

[28] J. Yang, B. Xia, Y. Shang, W. Huang, and C.C. Mi, "Adaptive State-of-Charge Estimation Based on a Split Battery Model for Electric Vehicle Applications," IEEE Transactions on Vehicular Technology, vol. 66, pp. 10889-10898, 2017.

[29] K. Lee, M. Dai, and C. Chuang, "Temperature-Compensated Model for Lithium-Ion Polymer Batteries With Extended Kalman Filter State-of-Charge Estimation for an Implantable Charger," IEEE Transactions on Industrial Electronics, vol. 65, pp. 589-596, 2018.

[30] L. Zheng, J. Zhu, D.D.-C. Lu, G. Wang, and T. He, "Incremental capacity analysis and differential voltage analysis based state of charge and capacity estimation for lithium-ion batteries," Energy, vol. 150, pp. 759-769, 2018.

[31] X. Li, Z. Wang, and L. Zhang, "Co-estimation of capacity and state-of-charge for lithium-ion batteries in electric vehicles," Energy, vol. 174, pp. 33-44, 2019. 
[32] X. Wu, X. Li, and J. Du, "State of Charge Estimation of Lithium-Ion Batteries Over Wide Temperature Range Using Unscented Kalman Filter," IEEE Access, vol. 6, pp. 41993-42003, 2018.

[33] Q. Song, Y. Mi, and W. Lai, "A Novel Variable Forgetting Factor Recursive Least Square Algorithm to Improve the Anti-Interference Ability of Battery Model Parameters Identification," IEEE Access, vol. 7, pp. 61548-61557, 2019.

[34] R. Xiong, F. Sun, H. He, and T.D. Nguyen, "A data-driven adaptive state of charge and power capability joint estimator of lithium-ion polymer battery used in electric vehicles," Energy, vol. 63, pp. 295-308, 2013.

[35] Z. Wei, C. Zou, F. Leng, B.H. Soong, and K. Tseng, "Online Model Identification and State-of-Charge Estimate for Lithium-Ion Battery With a Recursive Total Least Squares-Based Observer," IEEE Transactions on Industrial Electronics, vol. 65, pp. 1336-1346, 2018.

[36] Z. Chen, C.C. Mi, Y. Fu, J. Xu, and X. Gong, "Online battery state of health estimation based on Genetic Algorithm for electric and hybrid vehicle applications," Journal of Power Sources, vol. 240, pp. 184-192, 2013.

[37] Z. Chen, X. Shu, M. Sun, J. Shen, and R. Xiao, "Charging strategy design of lithium-ion batteries for energy loss minimization based on minimum principle," in 2017 IEEE Transportation Electrification Conference and Expo, Asia-Pacific (ITEC Asia-Pacific), 2017, pp. 1-6, 2017.

[38] Z. Chen, X. Shu, R. Xiao, W. Yan, Y. Liu, and J. Shen, "Optimal charging strategy design for lithium-ion batteries considering minimization of temperature rise and energy loss," International Journal of Energy Research, vol. 43, pp. 4344-4358, 2019.

[39] D. Yang, Y. Wang, R. Pan, R. Chen, and Z. Chen, "State-of-health estimation for the lithium-ion battery based on support vector regression," Applied Energy, vol. 227, pp. 273-283, 2018.

[40] Z. Chen, M. Sun, X. Shu, R. Xiao, and J. Shen, "Online State of Health Estimation for Lithium-Ion Batteries Based on Support Vector Machine," Applied Sciences, vol. 8, p. 925, 2018.

[41] Z. Chen, X. Xia, M. Sun, J. Shen, and R. Xiao, "State of Health Estimation of Lithium-Ion Batteries Based on Fixed Size LS-SVM," in 2018 IEEE Vehicle Power and Propulsion Conference (VPPC), 2018, pp. 1-6, 2018.

[42] W. An-na, Z. Yue, H. Yun-tao, and L.I. Yun-lu, "A novel construction of SVM compound kernel function," in 2010 International Conference on Logistics Systems and Intelligent Management (ICLSIM), 2010, pp. 1462-1465, 2010.

[43] Y. Deng, H. Ying, J. E, H. Zhu, K. Wei, J. Chen, F. Zhang, and G. Liao, "Feature parameter extraction and intelligent estimation of the State-of-Health of lithium-ion batteries," Energy, vol. 176, pp. 91-102, 2019.

[44] A. Eddahech, O. Briat, and J.-M. Vinassa, "Determination of lithium-ion battery state-of-health based on constantvoltage charge phase," Journal of Power Sources, vol. 258, pp. 218-227, 2014. 